\title{
Ergogenic and psychological effects of synchronous music during circuit-type exercise
}

C I Karageorghis (Contact Author), D L Priest, L S Williams, R M Hirani, K M Lannon and B J Bates

School of Sport \& Education, Brunel University, Uxbridge, Middlesex UB8 3PH, UK

Contact: costas.karageorghis@brunel.ac.uk

\section{Abstract \\ Objectives}

Motivational music when synchronized with movement has been found to improve performance in anaerobic and aerobic endurance tasks, although gender differences pertaining to the potential benefits of such music have seldom been investigated. The present study addresses the psychological and ergogenic effects of synchronous music during circuit-type exercise.

Design

A mixed-model design was employed in which there was a within-subjects factor (two experimental conditions and a control) and a between-subjects factor (gender).

Methods

Participants $(N=26)$ performed six circuit-type exercises under each of three synchronous conditions: motivational music, motivationally-neutral (oudeterous) music, and a metronome control. Dependent measures comprised anaerobic endurance, which was assessed using the number of repetitions performed prior to the failure to maintain synchronicity, and post-task affect, which was assessed using Hardy and Rejeski's (1989) Feeling Scale. Mixed-model 3 (Condition) $\times 2$ (Gender) ANOVAs, ANCOVAs, and MANOVA were used to analyze the data.

Results

Synchronous music did not elicit significant $(p<.05)$ ergogenic or psychological effects in isolation; rather, significant $(p<.05)$ Condition $\times$ Gender interaction effects emerged for both total repetitions and mean affect scores. Women and men showed differential affective responses to synchronous music and men responded more positively than women to metronomic regulation of their movements. Women derived the greatest overall benefit from both music conditions.

\section{Conclusions}

Men may place greater emphasis on the metronomic regulation of movement than the remaining, extra-rhythmical, musical qualities. Men and women appear to exhibit differential responses in terms of affective responses to synchronous music.

Keywords: Anaerobic endurance; Affect; Gender differences; Pacemaker; Rhythm; Tempo 


\section{Introduction}

One of the main dichotomies in music-related research in a sport and exercise context concerns the application of asynchronous vs. synchronous music (Karageorghis et al., 2009). Asynchronous music is played in the background to make the environment more pleasurable and there is no conscious synchronization between one's movement patterns and musical tempo or meter (Karageorghis and Terry, 1997); tempo is the "speed" of a piece of music as measured in beats per minute (bpm) whereas the term meter relates to how the listener perceives or "feels" the beat. Contrastingly, synchronous music is typified by the use of the rhythmic or temporal aspects of music as a type of metronome that regulates movement patterns (Anshel and Marisi, 1978; Hayakawa et al., 2000). For example, in synchronized swimming, the athletes strive to keep their aquatic dance routine in perfect time with the accompanying music.

Learning about the contingencies of music use is important in terms of harnessing its potential ergogenic (work enhancing), psychological, and psychophysical effects. Music has an ergogenic effect when it improves athletic/exercise performance by either delaying fatigue or increasing work capacity. Typically this results in higher-thanexpected levels of endurance, power, productivity, or strength (Atkinson et al., 2004; Elliott et al., 2005; Karageorghis et al., 2009; Lee and Clough, 2006; and Simpson and Karageorghis, 2006). Psychological effects refer to how music precipitates affective, (feelings of pleasure or displeasure), cognitive (thought processes), and behavioural responses. In the exercise context, the psychophysical effects of music concern the psychological perception of physical effort most often measured using ratings of perceived exertion (RPE; e.g., Hayakawa et al., 2000; and Karageorghis et al., 2009). The present study focuses on ergogenic and psychological effects.

Researchers have highlighted the natural predisposition of humans to respond to the rhythmical qualities of music (e.g., Karageorghis et al., 1999; and Large, 2000); musical rhythm can replicate natural movement-based rhythms. Large noted that while listening "We clap our hands, snap our fingers, stomp our feet, sway, dance or in some way coordinate our movements with the temporal structure that we perceive in musical events" (p. 528). This tendency sometimes results in synchronization between the tempo or speed of music and an athlete's movement patterns. A much-cited example from sport concerns the well-known Ethiopian distance runner Haile Gebrselassie who, in February 1998, smashed the indoor world record for $2000 \mathrm{~m}$ while synchronizing his stride rate to the rhythmical pop song Scatman, which was played over loudspeakers.

Despite the intuitive appeal of the rhythm response concept, very few studies in the sport and exercise psychology literature have investigated the impact of synchronous music. Nonetheless, ergogenic effects have been demonstrated in treadmill walking (Karageorghis et al., 2009), bench stepping (Hayakawa et al., 2000), cycle ergometry (Anshel \& Marisi, 1978), and 400 m running (Simpson \& Karageorghis, 2006).

Synchronization of movement to musical rhythm is a form of auditory-motor synchronization in which the actor (e.g., the athlete or dancer) and the auditory stimuli are "oscillators" which generate their own rhythms (Roerdink, 2008, p. 13).

Synchronization is characterized by an oscillation at a common frequency between two oscillators. The degree of synchronization is dependent on the strength of the interaction between the two oscillators (coupling strength) and the initial frequency mismatch between them. The actor can adjust his/her work rate to the tempo set by the auditory rhythm through detuning the frequency of their oscillation. Accordingly, in auditory- 
motor synchronization, the coupling is informational - because acoustic stimuli are processed by the actor's auditory system - and unidirectional in nature because only the work rate can be adjusted to changes in beats per minute and not the converse (Roerdink, 2008 p. 13).

There is a neuropsychological explanation for the proposed benefits of synchronous music during exercise and physical activity. The supplementary motor area of the brain plays an important role in both the perception of musical rhythm and the rhythmic ordering of motor tasks (Zatorre, Halpern, Perry, Meyer, and Evans, 1996). Indeed, Wilson (1986) referred to a pacemaker in the brain that is thought to regulate temporal functioning. Such a mechanism may serve to coordinate the afferent neural stimulus of music with a reciprocally-distributed efferent stimulus (i.e., physical movement). Along similar lines, Clynes and Walker (1982) highlighted a property of the central nervous system called time form printing; the propensity to execute repetitive patterns of movement with only the initial command requiring specific attention. Thus, once the shape and rate of the pattern have been established, effort can be directed elsewhere and movement efficiency enhanced.

An early study compared synchronous and asynchronous music using a cycle ergometer endurance task (Anshel and Marisi, 1978). Synchronous music elicited longer endurance than either asynchronous music or a no-music control (Cohen's $d=.6$ for synchronous vs. control). The music was chosen in an arbitrary manner and female participants severely underperformed in terms of endurance despite working at the same relative intensity as their male counterparts. The authors explained the females' underperformance with reference to the phenomenon of social influence. Specifically, females may not wish to exert themselves maximally in the presence of a male experimenter. In a related study, Hayakawa et al. (2000) examined how synchronous and asynchronous music influenced mood during step-aerobics classes. Participants reported more positive moods in response to synchronous music; however, it remains unclear whether such purported benefits were associated with the music itself or the physiological demands of the class (e.g., thermoregulation or oxygen uptake).

More recently, Karageorghis et al. (2009) examined the effects of motivational synchronous music and oudeterous (neutral in motivational terms; see Karageorghis et al., 1999) synchronous music on time to exhaustion, RPE, in-task affect, and exerciseinduced feeling states during treadmill walking at $75 \%$ of maximal heart rate reserve. The authors hypothesized that the motivational synchronous music condition would yield the most positive outcomes followed by the oudeterous condition. It was also expected that RPE during the early stages of the task would be lower in the motivational music condition when compared to the oudeterous condition and a no-music control. Although the RPE results did not achieve statistical significance (possibly owing to low observed power of .43), the motivational music enhanced in-task affect throughout the exercise bout and even at 2 min prior to voluntary exhaustion. This condition also yielded a $15 \%$ increase in endurance over control and a $6 \%$ increase over oudeterous music.

In addition to the benefits associated with asynchronous music detailed within the conceptual framework of Karageorghis et al. (1999), it has been proposed that the synchronous use of music reduces the metabolic cost of exercise by promoting greater neuromuscular or metabolic efficiency (Karageorghis, 2008; and Roerdink, 2008, pp. 220-221). A regular kinetic pattern may require less energy to replicate owing to the 
absence of minute adjustments (see Smoll and Schultz, 1982) and a greater relaxation which comes from the precise expectancy of the forthcoming movement.

Until fairly recently, there had been scant research into the effects of synchronous music on anaerobic endurance performance. Simpson and Karageorghis (2006) sought to address this gap in the literature by examining the effects of synchronous music during $400 \mathrm{~m}$ track running using an externally valid, race-like protocol. Their findings showed that both motivational and oudeterous music conditions elicited faster times than a nomusic control condition and that the times associated with the two experimental conditions did not differ. This latter finding indicates that the motivational qualities of music may not be of critical importance when it is used synchronously for an anaerobic endurance task; a notion that is entirely consistent with Rejeski's (1985) parallel processing theory and Tenenbaum's (2001) exercise intensity-attention linkage model. Nonetheless, there is considerable scope for further investigation of the ergogenic and psychological effects of music in anaerobic and rhythmical sports (e.g., canoeing/kayaking, cycling and rowing) and in repetitive training activities (e.g., circuit training and resistance training).

The limited evidence suggests that synchronous music can be applied to aerobic and anaerobic endurance performance among nonelite athletes to produce ergogenic and positive psychological effects. Recently, theorists have speculated that synchronous music applied to submaximal repetitive activity might result in an efficiency gain wherein work rate is maintained at lower metabolic cost (e.g., Karageorghis and Terry, 2009). Nonetheless, there is insufficient research underlying the use of synchronous music, especially with a specific focus on gender differences, rendering this a particularly fruitful area for future research.

\section{The moderating influence of gender}

There is scant research evidence or indeed theoretical dialogue on the differential response of the genders to music in an exercise context. Given women's greater exposure to dance and movement-to-music during their formative years (see Shen, Chen, Scrabis, and Tolley, 2003) and the greater likelihood of women dancing at social events (Crowther and Durkin, 1982) it may be conceivable that females demonstrate a more pronounced response to synchronous music. However, such assertions are rather tenuous in nature. Recent work that has examined the moderating influence of gender has not found differences in musical reactivity between women and men (Karageorghis et al., 2006; Karageorghis et al., 2008 and Karageorghis et al., 2009). Nevertheless, there is a pronounced choreographic element to exercise modes which are popular with female participants in particular (e.g., step aerobics). Hence, the absence of gender moderation has been explained in terms of the lack of a choreographic element in the tasks used (Karageorghis et al., 2008). Indeed, the experimental tasks have typically involved long-duration, repetitive movements such as cycle ergometry or treadmill walking (Anshel and Marisi, 1978; and Karageorghis et al., 2009).

The main purpose of the present study was to investigate the effect of synchronous music (motivational and oudeterous) and an audible-metronome control on anaerobic endurance and affect using a series of standardized circuit-type exercises. It was hypothesized that the motivational music condition would yield greater anaerobic endurance and the most positive psychological outcomes followed by the oudeterous condition and the metronome control condition. Motivational or stimulative music has led to improved anaerobic performance in a variety of tasks (see e.g., Karageorghis et al., 
1996; and Simpson and Karageorghis, 2006) as theoretically predicted (see Karageorghis and Terry, 1997; and Karageorghis et al., 1999). Secondly, it was also hypothesized that females would derive greater benefit than males from both the motivational and oudeterous music conditions in terms of anaerobic endurance. Based on previous research findings (Karageorghis et al., 2008; and Karageorghis et al., 2009), no gender difference was expected to emerge for affect.

\section{Method}

\section{Stage 1: music selection}

\section{Participants and procedure}

A sample of 150 volunteer, third-year sports science undergraduates who were white and of UK/Irish origin, was used to identify a pool of artists for use in the experimental protocol of Stage 2. It was intended that these students would match the profile of the intended pool of experimental participants both in terms of age and socio-cultural background (cf. Karageorghis and Terry, 1997; and Karageorghis et al., 2006). Ethical clearance was obtained for each stage of the present study from the Brunel University Ethics Committee and participants were required to provide written informed consent.

Participants were asked to record their three favourite up-tempo pieces of music for circuit training-type exercises on a response sheet in hierarchical order. They were also asked to record three pieces of up-tempo music that would be suitable for circuit training but would not count among their favourite selections. Subsequently, the tracks were classified into music idioms by the researchers (e.g., classical, jazz, latin, pop, reggae, etc.) and the 12 highest-rated pop tracks from each list (favourite and suitable) were subjected to further testing. These 24 tracks also had to fit within the tempo range 112$128 \mathrm{bpm}$ to facilitate the synchronous nature of the experimental tasks in which movement rate was standardized at $120 \mathrm{bpm}$. It was necessary to digitally alter the tempi of some of the tracks and have either one or two repetitions of each exercise conducted in each musical bar (four crotchet [ $\downarrow]$ beats).

Tracks from other musical idioms such as jazz, latin, and rock were discarded to ensure consistency across terms of musical idiom in the experimental conditions. It should be noted that the complex, syncopated rhythms of jazz and latin are generally unsuitable for synchronous exercise. One track from the pop idiom was also discarded as it was deemed through piloting that it did not facilitate synchronous exercise owing to its uneven rhythmic structure. The two sets of 12 tracks were rated to assess their motivational qualities using the Brunel Music Rating Inventory-2 (BMRI-2: Karageorghis, Priest, et al., 2006). This procedure ensured that there would be homogeneity in terms of the motivational qualities of the music in each of the motivational and oudeterous music conditions and that the tracks met the criteria relating to the motivational qualities of music (Karageorghis et al., 2006; and Karageorghis et al., 1999).

A panel of nine undergraduate sports science students (mean age $=21.0$ years, $S D=1.0$ years) rated 24 tracks using the BMRI-2. This panel matched the profile of the intended pool of experimental participants in terms of age and socio-cultural background. They rated the motivational qualities of each track with reference to a circuit-type task in accordance with the instructions of Karageorghis, Priest, et al. (2006). Nine tracks from each of the two original lists of 12 with similar motivational qualities were selected by the researchers to constitute the motivational and oudeterous 
experimental conditions. As previously indicated, in their original form, the tracks played in the tempo range $112-128 \mathrm{bpm}$, however tempo was standardized at $120 \mathrm{bpm}$ for the two synchronous music conditions using a computer software package (Virtual DJ v. 3.3; Atomic Productions, Paris, France). Copyright permission was requested from the music publishers to record the tracks for research purposes; full details are available upon request.

\section{Stage 2: experimental investigation}

\section{Power analysis}

A power analysis to establish appropriate sample size based on the large effect size (partial $\eta^{2}=.24$ ) of Simpson and Karageorghis (2006) for the impact of synchronous music on anaerobic endurance, indicated that approximately 20 participants would be required. An even split across the genders was aimed for while an extra three participants in each group protected the study against the possibility of experimental drop-out and multivariate outliers (i.e., 26 experimental participants in total). There were no previous data concerning synchronous exercise tasks from which to calculate appropriate power for the Condition $\times$ Gender interaction; accordingly this part of the analysis was exploratory in nature.

\section{Participants}

The 26 participants comprised 13 women (mean age $=18.9$ years, $S D=.8$ years) and 13 men (mean age $=18.7$ years, $S D=.8$ years) from the body of first-year sports science undergraduates at Brunel University. Participants were white, brought up in the United Kingdom and homogeneous in terms of their age and socio-cultural background. They were drawn from sports that require circuit-type and resistance training as part of their conditioning programme but were not elite athletes (e.g., student internationals or senior internationals). An inducement was provided to recruit participants: their names were entered into a draw for an item of sports apparel, with separate draws conducted for women and men.

\section{Apparatus and measures}

A number of mats, barbells, and benches were used for testing along with a CD player (EXP2460; Philips Electronics, Beijing, China) and a decibel meter (GA 102 Sound Level Meter Type 1; Castle Associates, Scarborough, UK) to standardize music intensity at 75 dBA. Four speakers were used (SRS-M30; Sony, Bangkok, Thailand) to enable the sound to be heard at a similar intensity at each circuit station (see Figure 1). Two digital cameras (EX-260 Exilim Optical; Casio, Beijing, China) were used to film each trial so that the number of repetitions performed could be verified post hoc. A handheld stopwatch (Nike Triax 26; Nike, Eugene, OR) was used to time the recovery period between circuit exercises. 


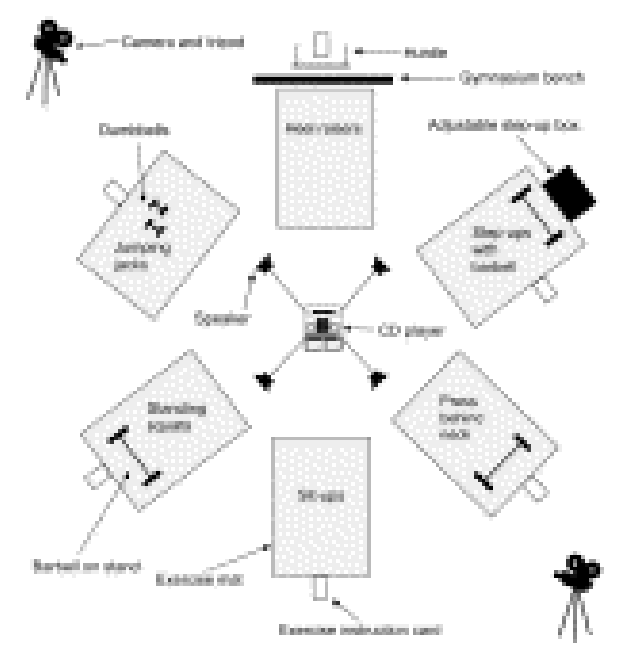

Figure 1: Schematic of experimental set-up

In-task affect was assessed by use of Hardy and Rejeski's (1989) bipolar Feeling Scale (FS) which was designed specifically for an exercise context. The FS is an 11-point, single-item scale which ranges from +5 (very good) to -5 (very bad) and has a midpoint of 0 (neutral). The developers of the FS demonstrated its validity through a series of three studies: The single dimension of affect (positive vs. negative) was validated using discriminant function analysis. Specifically, the positive affect subscale of the Multiple Affective Adjective Checklist was used to discriminate with $95.2 \%$ accuracy between two matched groups of participants who were instructed to check adjectives associated with either "good" or "bad" feeling states. In subsequent studies, the FS demonstrated negative correlations with HR $(r=-.70 ; p<.05)$, respiratory rate $(r=-.62 ; p<.05)$, and oxygen uptake $(r=-.69 ; p<.05)$ at a variety of exercise intensities.

\section{Pre-test and habituation trials}

Participants had their anaerobic endurance tested using the six exercises that were administered during the experimental trials (press behind neck, sit-ups, standing squats with barbell, jumping jacks with dumbbells, heel raisers on a bench, and step-ups) to enable this variable to be factored out of the analysis as a covariate. Specifically, they performed each exercise at a standardized movement tempo of $120 \mathrm{bpm}$, which was visually cued by an experimenter using mirroring of the movement. Participants continued with each exercise until they could no longer maintain the preordained work rate for four consecutive bars, after which point they had a 3 min recovery before attempting the next exercise.

Following the pre-test, participants were further habituated to the circuit-type exercises so as to ensure their familiarity with good technique and their ability to complete repetitions in strict time with musical tempo. They spent approximately 20 min conducting the exercises during the habituation trial, during which the experimental protocol was explained thoroughly. Thus the experimenters met each experimental participant on five occasions: once to test their anaerobic endurance, once for a habituation trial, and three times for the experimental trials.

\section{Experimental trials}


Participants were exposed to two experimental conditions (motivational and oudeterous synchronous music) and a metronome-only control condition. The order in which they were requested to complete the exercises was counterbalanced across participants to guard against any potential order effects (Harris, 2008, pp. 156-161). Participants were required to follow identical patterns of activity and diet with no other vigorous physical activity permitted prior to each trial. Further, they were not permitted to eat a meal within $2 \mathrm{~h}$ prior to testing. Participants engaged in the trial in groups of three in the presence of a same-sex experimenter (cf. Anshel and Marisi, 1978); however no two participants faced each other during testing and there was a gap of one exercise station between them (see Figure 1).

Music intensity was standardized at $75 \mathrm{dBA}$ for each of the 18 tracks by use of the decibel meter. This is an upper-level intensity that would typify most exercise facilities but still lie within safe limits from an audiological perspective (Alessio and Hutchinson, 1991). In-task affect was assessed at the end of each of the six exercises in the circuit. Participants were given a 3 min rest period at the point that synchronous movement could not be maintained for four consecutive musical bars or measures (16 crotchet beats). Piloting of the protocol indicated that in $3 \mathrm{~min}$, participants' heart rates were likely to return close to resting levels. Water was offered to participants during this rest period. The order in which each participant progressed through the test protocol was not altered from trial to trial in order to facilitate meaningful comparison of the dependent variables. The dependent measure of work completed consisted of the number of repetitions for each exercise and the sum of repetitions from each of the six exercises.

\section{Data analysis}

Data were screened for univariate outliers using z scores $> \pm 3.29$ and for multivariate outliers using the Mahalanobis distance method with $p<.001$ (Tabachnick and Fidell, 2007, pp. 224-227). There were multiple dependent variables (number of repetitions for each exercise, total number of repetitions across exercises, in-task affect for each exercise, mean in-task affect), a single covariate (anaerobic endurance) used for analyses of the repetitions only, and two independent variables: condition (motivational synchronous music, oudeterous synchronous music, and a metronome control) and gender. Thus, following checks to ensure that the data were suitable for parametric analysis, mixed-model $3 \times 2$ (Condition $\times$ Gender) ANCOVAs/ANOVAs were computed with Bonferroni adjustments. Pairwise comparisons, also with Bonferroni adjustments, were used to establish where differences lay. A mixed-model MANOVA was used to analyze the affect data for the six exercises. Mauchly's test was used to check the assumption of sphericity and Greenhouse-Geisser adjustments made to $F$ tests where necessary.

\section{Results}

For Stage 1, BMRI-2 scores for the motivational and oudeterous synchronous music conditions were compared using a paired-samples $t$ test which, as expected, indicated a significant difference, $t_{8}=41.60, p<.001$, with the motivational condition yielding the highest mean $(M$ Mot. $=30.90$ vs. $M$ Oud. $=22.11$ ). In Stage 2 , data screening revealed one case with multiple univariate outliers that was removed from the dataset prior to further analyses. Tests of the distributional properties of the data in each cell of each analysis revealed violations of normality in 14 out of 126 cells $(11.1 \%$; nine at $p<.05$, three at $p<.01$, and two at $p<.001$ ). However, MANOVA and ANOVA/ANCOVA are 
sufficiently robust to withstand such minor deviations from normality (Keppel and Wickens, 2004).

\section{Interaction effects}

The interaction of Condition $\times$ Gender for total repetitions was significant, $F(2$, $44)=4.66, p<.05, \eta_{p}^{2}=.18$, and accounted for $18 \%$ of the variance, which represents a large effect. The significant interaction was caused by differences in how women and men responded to the metronome control condition; while the female participants' performance did not show any degradation when the oudeterous condition $(M=267.29$, $S E=9.36)$ was compared to the control $(M=260.74, S E=14.05)$, the male participants performed better in the control condition $(M=247.60, S E=9.75$ vs. $M=278.02, S E=14.63)$. Also, a visual inspection revealed that the men exhibited greater variability in their scores (see T-bar SDs in Figure 2) when compared to women in each of the three conditions.

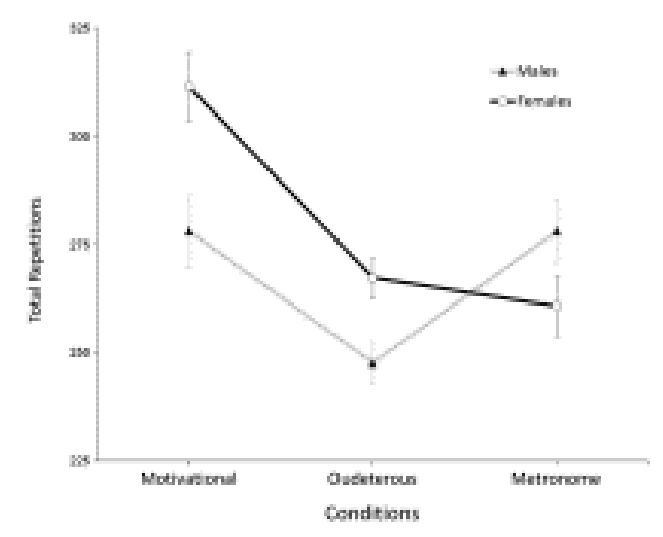

Figure 2: Music condition $\times$ gender interaction for total repetitions $(p<.05)$. Note. The plotted means are not covariate adjusted. The error bars denote the magnitude of standard error.

The interaction of Condition $\times$ Gender for individual exercise repetitions was significant only for step-ups, and exhibited a large effect, $F(2,44)=5.75, p<.008, \eta_{p}^{2}=.21$. The pattern of differences revealed significant degradation in performance among women from motivational to oudeterous to control conditions, whereas the men's performance remained relatively stable across conditions (see Table 1 ). The interaction for mean affect (across the six exercises) of Condition $\times$ Gender was significant, $F(2,46)=5.16$, $p<.05, \eta_{\mathrm{p}}^{2}=.18$, and accounted for $18 \%$ of the variance; a large effect. Inspection of pairwise comparisons revealed that female participants exhibited less negative affect than males in both music conditions (motivational female $M=-.06, S E=.26$ vs. male $M=-.64, S E=.27$; oudeterous female $M=-.01, S E=.28$ vs. male $M=-.83$, $S E=.30)$. In the control condition, the males exhibited less negative affect than females (female $M=-.24, S E=.25$ vs. male $M=-.15, S E=.26$ ) although this segment of the interaction did not quite reach significance $(p>.05)$. In the MANOVA for affect across individual exercises, the Condition $\times$ Gender interaction was nonsignificant, Pillai's Trace $=.47, F(12,12)=.87, p>.05, \eta_{\mathrm{p}} 2=.47$, but accounted for a very large amount of explained variance $(47 \%)$. 
Table 1: Descriptive statistics (means and standard deviations) for music condition and gender

\begin{tabular}{|c|c|c|c|c|c|c|c|c|c|c|c|c|c|c|}
\hline \multirow{2}{*}{$\begin{array}{l}\text { Condition/ } \\
\text { gender }\end{array}$} & \multicolumn{2}{|c|}{ Press behind neck } & \multicolumn{2}{|l|}{ Sit-ups } & \multicolumn{2}{|c|}{ Standing squats } & \multicolumn{2}{|c|}{ Jumping jacks } & \multicolumn{2}{|l|}{ Heel raisers } & \multicolumn{2}{|c|}{ Step-ups } & \multirow[t]{2}{*}{ Total reps } & \multirow{2}{*}{$\begin{array}{l}\text { Mean } \\
\text { affect }\end{array}$} \\
\hline & Reps & Affect & Reps & Affect & Reps & Affect & Reps & Affect & Reps & Affect & Reps & Affect & & \\
\hline \multicolumn{15}{|l|}{ Motivational } \\
\hline Females & $\begin{array}{l}21.15 \\
(6.12)\end{array}$ & \begin{tabular}{|l|}
.15 \\
$(-1.57)$
\end{tabular} & $\begin{array}{l}50.23 \\
(23.69)\end{array}$ & $.15(1.34)$ & $\begin{array}{l}47.85 \\
(21.43)\end{array}$ & $-.15(.90)$ & $\begin{array}{l}85.31 \\
(18.13)\end{array}$ & $.15(.90)$ & $\begin{array}{l}58.35 \\
(17.29)\end{array}$ & $-.54(1.27)$ & $\begin{array}{l}58.46 \\
(19.21)\end{array}$ & $.15(1.52)$ & $\begin{array}{l}309.23 \\
(81.19)\end{array}$ & $-.06(.93)$ \\
\hline Males & $\begin{array}{l}20.25 \\
(2.42)\end{array}$ & $-.5(-.9)$ & $\begin{array}{l}39.83 \\
(14.10)\end{array}$ & $-.83(1.34)$ & $\begin{array}{l}61.00 \\
(19.45)\end{array}$ & \begin{tabular}{|l|}
-.25 \\
$(1.48)$
\end{tabular} & $\begin{array}{l}70.50 \\
(16.81)\end{array}$ & \begin{tabular}{|l|}
.33 \\
$(1.37)$
\end{tabular} & $\begin{array}{l}48.67 \\
(12.34)\end{array}$ & $\begin{array}{l}-1.42 \\
(1.24)\end{array}$ & $\begin{array}{l}41.08 \\
(16.10)\end{array}$ & $\begin{array}{l}-.50 \\
(1.45)\end{array}$ & $\begin{array}{l}280.50 \\
(44.60)\end{array}$ & $-.64(.92)$ \\
\hline Overall & $\begin{array}{l}20.72 \\
(4.65)\end{array}$ & $\begin{array}{l}-.32 \\
(-1.28)\end{array}$ & $\begin{array}{l}45.24 \\
(20.00)\end{array}$ & $-.32(1.41)$ & $\begin{array}{l}54.16 \\
(21.17)\end{array}$ & $\begin{array}{l}-.20 \\
(1.19)\end{array}$ & $\begin{array}{l}78.20 \\
(18.73)\end{array}$ & $\begin{array}{l}-.08 \\
(1.15)\end{array}$ & $\begin{array}{l}53.72 \\
(15.62)\end{array}$ & $-.96(1.31)$ & $\begin{array}{l}50.12 \\
(19.54)\end{array}$ & $\begin{array}{l}-.16 \\
(1.49)\end{array}$ & $\begin{array}{l}295.44 \\
(66.50)\end{array}$ & $-.34(.95)$ \\
\hline \multicolumn{15}{|l|}{ Oudeterous } \\
\hline Females & $\begin{array}{l}19.08 \\
(5.36)\end{array}$ & $.31(-1.93)$ & $\begin{array}{l}43.15 \\
(16.57)\end{array}$ & $.46(2.03)$ & $\begin{array}{l}40.38 \\
(13.33)\end{array}$ & \begin{tabular}{|l}
-.08 \\
$(1.44)$
\end{tabular} & $\begin{array}{l}72.00 \\
(15.76)\end{array}$ & \begin{tabular}{|l|}
-.30 \\
$(1.03)$
\end{tabular} & $\begin{array}{l}45.30 \\
(11.81)\end{array}$ & $-.23(2.01)$ & $\begin{array}{l}49.92 \\
(16.40)\end{array}$ & $\begin{array}{l}-.08 \\
(1.67)\end{array}$ & $\begin{array}{l}265.30 \\
(46.91)\end{array}$ & $.01(1.22)$ \\
\hline Males & $\begin{array}{l}19.08 \\
(2.43)\end{array}$ & $-.67(-1.5)$ & $\begin{array}{l}37.42 \\
(12.89)\end{array}$ & $\begin{array}{l}-1.08 \\
(1.08)\end{array}$ & $\begin{array}{l}47.41 \\
(12.27)\end{array}$ & $\begin{array}{l}-.58 \\
(1.31)\end{array}$ & $\begin{array}{l}60.33 \\
(10.13)\end{array}$ & $\begin{array}{l}-.25 \\
(1.21)\end{array}$ & $43.67(9.31)$ & $\begin{array}{l}-1.58 \\
(1.16)\end{array}$ & $\begin{array}{l}42.00 \\
(13.49)\end{array}$ & $\begin{array}{l}-.83 \\
(1.40)\end{array}$ & $\begin{array}{l}249.75 \\
(42.63)\end{array}$ & $-.83(.77)$ \\
\hline Overall & $\begin{array}{l}19.08 \\
(4.13)\end{array}$ & $\begin{array}{l}-.16 \\
(-1.77)\end{array}$ & $\begin{array}{l}40.40 \\
(14.90)\end{array}$ & $-.28(1.79)$ & $\begin{array}{l}43.76 \\
(13.06)\end{array}$ & $\begin{array}{l}-.32 \\
(1.38)\end{array}$ & $\begin{array}{l}66.40 \\
(14.37)\end{array}$ & \begin{tabular}{|l|}
-.28 \\
$(1.10)$
\end{tabular} & $\begin{array}{l}44.52 \\
(10.49)\end{array}$ & $-.88(1.76)$ & $\begin{array}{l}46.12 \\
(15.31)\end{array}$ & \begin{tabular}{|l|}
-.44 \\
$(1.53)$
\end{tabular} & $\begin{array}{l}257.84 \\
(44.68)\end{array}$ & $\begin{array}{l}-.39 \\
(1.09)\end{array}$ \\
\hline \multicolumn{15}{|l|}{ Control } \\
\hline Females & $\begin{array}{l}18.69 \\
(4.94)\end{array}$ & $.23(-1.59)$ & $\begin{array}{l}40.77 \\
(18.61)\end{array}$ & $.08(1.71)$ & $\begin{array}{l}43.69 \\
(18.92)\end{array}$ & $.15(1.28)$ & $\begin{array}{l}70.54 \\
(15.11)\end{array}$ & \begin{tabular}{|l|}
-.77 \\
$(1.17)$
\end{tabular} & $\begin{array}{l}49.46 \\
(15.37)\end{array}$ & $-.54(1.20)$ & $\begin{array}{l}42.15 \\
(15.70)\end{array}$ & \begin{tabular}{|l}
-.62 \\
$(1.39)$
\end{tabular} & $\begin{array}{l}259.38 \\
(58.69)\end{array}$ & $\begin{array}{l}-.24 \\
(1.00)\end{array}$ \\
\hline Males & $\begin{array}{l}18.75 \\
(3.36)\end{array}$ & $\begin{array}{l}-.25 \\
(-1.06)\end{array}$ & $\begin{array}{l}43.00 \\
(22.16)\end{array}$ & $-.25(.75)$ & $\begin{array}{l}51.75 \\
(15.30)\end{array}$ & $.33(1.67)$ & $\begin{array}{l}66.17 \\
(15.10)\end{array}$ & $.08(1.08)$ & $51.83(5.89)$ & $-.50(1.09)$ & $\begin{array}{l}39.67 \\
(14.49)\end{array}$ & $\begin{array}{l}-.33 \\
(1.50)\end{array}$ & $\begin{array}{l}279.50 \\
(47.69)\end{array}$ & $-.15(.77)$ \\
\hline Overall & $\begin{array}{l}18.72 \\
(4.17)\end{array}$ & $0(-1.35)$ & $\begin{array}{l}41.84 \\
(19.98)\end{array}$ & $-.08(1.32)$ & $\begin{array}{l}47.56 \\
(14.41)\end{array}$ & $.24(1.45)$ & $\begin{array}{l}68.44 \\
(14.96)\end{array}$ & $\begin{array}{l}-.36 \\
(1.18)\end{array}$ & $\begin{array}{l}50.60 \\
(11.64)\end{array}$ & $-.52(1.12)$ & $\begin{array}{l}40.96 \\
(14.87)\end{array}$ & $\begin{array}{l}-.48 \\
(1.42)\end{array}$ & $\begin{array}{l}269.04 \\
(53.58)\end{array}$ & $-.20(.88)$ \\
\hline
\end{tabular}

Note. The means and standard deviations displayed are not covariate adjusted. 


\section{Main effects}

For total repetitions, there were no significant main effects either for condition, $F(2$, $44)=.91, p>.05, \eta_{p}^{2}=.04$, or gender, $F(1,22)=.51, p>.05, \eta_{p}^{2}=.02$. For individual exercise repetitions, there were again no significant $(p<.008)$ main effects. For mean affect, there were no significant main effects for condition, $F(2,46)=1.06$, $p>.05, \eta_{\mathrm{p}}{ }^{2}=.08$, or gender, $F(1,23)=1.71, p>.05, \eta_{\mathrm{p}}{ }^{2}=.07$, and the MANOVA for affect scores in individual exercises was also nonsignificant for condition, Pillai's Trace $=.66, F(12,12)=1.90, p>.05, \eta_{p}{ }^{2}=.66$, and gender, Hotelling's Trace $=.28$, $F(6,18)=.84, p>.05, \eta_{\mathrm{p}}{ }^{2}=.22$. Nonetheless, both omnibus statistics were associated with large effect sizes. Notwithstanding the nonsignificance of the main effect for condition, the highest performance levels for both women and men were achieved in the motivational synchronous condition (see Table 1 and Figure 2).

\section{Discussion}

The main purpose of the present study was to investigate the influence of synchronous music on anaerobic endurance and affect using a range of standardized circuit-type exercises. A secondary purpose was to examine the possible moderating influence of gender in reactivity to synchronous music. It was hypothesized that the motivational music condition would yield greater anaerobic endurance and more positive psychological outcomes, followed by the oudeterous and then the metronome control conditions. While this general trend did emerge (see Table 1), the differences across conditions did not reach statistical significance $(p>.008)$; accordingly, the principal research hypothesis was refuted. The secondary hypothesis held that women would derive greater benefit than men from both music conditions in terms of anaerobic endurance scores. Although there was a significant interaction effect, it did not follow the hypothesized pattern in that men performed unexpectedly well in the control condition. For affect, the individual exercises did not elicit any significant $(p<.008)$

Condition $\times$ Gender interaction; nonetheless, a trend emerged wherein women appeared to derive greater benefit from the two music conditions (see Table 1 and Figure 2). This trend was magnified in the interaction for mean affect (across the six exercises) which did reach significance $(p<.05)$ and accounted for $18 \%$ of the variance. Collectively, the affect results lead us to refute the secondary research hypothesis as there were significant gender differences for mean affect. In sum, the results were not entirely as expected, however this lack of support for the research hypotheses should be considered in light of the study's novelty.

\section{Condition $\times$ Gender interactions}

The only individual exercise that yielded a significant interaction effect for repetitions was step-ups $\left(p<.008, \eta_{p}^{2}=.21\right.$; see Table 1$)$. Whereas women followed the expected pattern of detriments in performance from the motivational to the oudeterous to the control conditions, the men did not (see Table 1). This may have been due to the fact that stepping is a modality of exercise that has been popularized among women through exercise-to-music classes. Hence, the female participants may have had greater stepping experience or perceived stepping to be a more "feminine" activity.

There was a strong interaction effect for total repetitions performed $\left(\eta_{\rho}^{2}=.18\right.$; see Figure 2). In essence, both women and men followed the expected trend of producing fewer repetitions in the oudeterous condition than in the motivational condition. 
Nonetheless, whereas women performed fewest repetitions under the control condition, men performed as well as they had in the motivational condition. This result may indicate that men are more able to coordinate their movements to a regular beat in the absence of musical stimuli and that the coupling strength (Roerdink, 2008, p. 13) is reduced slightly in the presence of oudeterous music. Hence, men may place more importance than women on the "pacemaking" effect of a regular beat which runs contrary to the findings of earlier questionnaire-based research (Karageorghis et al., 1999; and Priest et al., 2004). However, these studies differed from the present work, not only in their descriptive as opposed to experimental nature, but also in their focus on the effects of rhythmical structure in a generic sense rather than metronomic regulation of movement. Moreover, it is conceivable that the men responded positively to the motivational music, while in the control condition, it was simply easier for them to focus on their movement rate. Accordingly, the equal levels of performance across these two conditions may well be associated with different antecedents.

Table 1 reveals greater variability in total repetition scores among women, particularly in the motivational music condition. This indicates that there may be larger individual differences among women in terms of their response to synchronous music during anaerobic activity. The present findings allude to the influence of a hidden variable such as rhythmic ability (Large, 2000); however gender differences in rhythmic ability have yet to be thoroughly investigated. Through observation, we might speculate that some women are more adept at keeping time than others, while men are relatively homogeneous in their rhythmic ability.

Several tentative reasons were advanced to support the prediction that women would derive more benefit from synchronous music; females experience greater exposure to dance music and movement-to-music during their formative years (Crowther and Durkin, 1982), and demonstrate a greater desire to engage in dance-related activity (Shen et al., 2003). It should be acknowledged that these arguments are speculative in nature and that further research is needed to clarify the actual causes of any gender differential. It was found that women and men derived similar benefit from the synchronous application of music. Notably, this outcome is concurrent with recent studies that have reported a lack of gender differential in reactivity to music (Karageorghis et al., 2006; Karageorghis et al., 2008; and Karageorghis et al., 2009). These studies were based around simple and repetitive motor tasks such as cycling or walking, which did not place great demands on the participants in terms of physical coordination.

With reference to affect, men experienced more negative feeling states than women in both music conditions. Interestingly, the opposite trend was evident in the control condition. In the domain of physical activity, the present results are quite novel. Relevant conceptual models (see Karageorghis and Terry, 2009) indicate that music can elicit positive affect during exercise and this has been demonstrated even when exercise is performed at a relatively high intensity (see e.g., Edworthy and Waring, 2006; and Karageorghis et al., 2009). Nonetheless, in the wider psycho-musicological literature, there is some evidence to suggest that women experience a stronger affective response to music (Hargreaves \& North, 2008, pp. 113-115).

Notably, multivariate analysis of individual exercises did not reveal significant $(p<.008)$ interaction effects. Nonetheless, $47 \%$ of the variance was explained by the music manipulation which indicates a meaningful effect. The declaration of statistical 
significance was possibly limited by discrepancies in the direction of the difference across individual exercises (see Table 1). Ostensibly, synchronous music affected different exercises in different ways. It should be noted that women did not perform better than the men in relative terms. Hence, the improved affect that women experienced did not translate into elevated performance levels. With reference to the control condition, it is probable that the female participants either experienced greater negative affect in response to the absence of the music, or that the male participants felt less negative because they actually performed better. A further explanation that merits consideration is that of self-presentation anxiety (Leary and Kowalski, 1995). It is possible that the women were more comfortable to be seen moving to the music as this conforms more closely to gender-related behavior in the exercise context (see Crowther and Durkin, 1982).

\section{Influence of synchronous music on anaerobic endurance}

The failure of the experimental conditions to influence performance is somewhat unexpected as previous studies have consistently demonstrated the ergogenic effects of synchronous music in activities such as $400 \mathrm{~m}$ running (Simpson and Karageorghis, 2006), and treadmill walking to exhaustion (Karageorghis et al., 2009). However, the tasks chosen for previous studies were not as demanding in terms of physical load or as reliant on motor skills as those used in the present study. Music is more likely to exert an ergogenic effect when there is the possibility for it to influence voluntary performance as in a gymnasium-type "workout" rather than a strictly-controlled exhaustive effort. In endurance-based activities, phenomena such as time form printing (Clynes and Walker, 1982) may more readily explain the ergogenic effects of synchronous music; whereas, in the present study, the movements performed may have required more conscious attention thus obviating any proposed gain in efficiency resulting from synchronicity (see Smoll and Schultz, 1982). Hence, the detuning required to reduce the initial frequency mismatch between the musical and physical oscillations may have proved too challenging, resulting in reduced coupling strength (Roerdink, 2008, p. 13).

\section{Influence of synchronous music on affect}

The music conditions per se did not influence affect which confounds the results of comparable studies (see e.g., Edworthy and Waring, 2006; Hayakawa et al., 2000; and Karageorghis et al., 2009) and conceptual predictions that motivational music improves affect (see Karageorghis and Terry, 2009). However, affect was measured immediately post-task rather than in-task, which might account for this difference. As well as measuring in-task affect, Karageorghis et al. assessed post-exercise feeling states and these did not show a significant $(p<.05)$ difference in response to analogous experimental conditions. The interaction effect reveals that synchronous music enhanced the feeling states of female participants only. Hence, the question of most interest is why this pattern did not apply to the male participants (see Table 1). It is plausible that the mode of exercise may have shaped the findings somewhat. Previous work has generally made use of simple motoric tasks of long-duration (see Anshel and Marisi, 1978; and Karageorghis et al., 2009) whereas the present study was based on anaerobic tasks that required some element of skill. Potentially, the affective response to music is different under these conditions. It is notable that, while the music conditions did not influence affect across the individual exercises, they did account for a very large percentage of variance explained (66\%). 


\section{Limitations of the present study}

It may have been that the tasks used in the present study were insufficiently complex for any gender difference to have emerged; for example, sit-ups constitute a gross motor skill which has little potential for stylistic interpretation. While the exercises used were not fully choreographed, they did represent a shift away from the repetitive endurance activities previously employed (e.g., Karageorghis et al., 2009) towards more complex movement patterns. Also, the use of active sportspersons may have precluded a gender effect; this is because the exercises used are staples within the sports training environment and this would have reduced the technical demands placed upon participants. Assessing performance in resistance exercises (such as jumping jacks and military press) across genders presents challenges in terms of equalizing load. Despite extensive piloting of the protocol, the present results suggest that, in some cases, specifying loads for male and female participants was problematic. Hence, resistance should perhaps be assigned on an individual basis as in previous studies that employed cycle ergometers or treadmills (Anshel and Marisi, 1978; Karageorghis et al.).

The concept of load also applies to repetitious exercises that rely on bodyweight rather than added resistance; for example, some individuals are able to complete more than 100 sit-ups whereas others might experience their own bodyweight as a near maximal load and struggle to complete even 10 repetitions with correct form. From a statistical standpoint, certain parts of the analysis were underpowered (e.g., affect omnibus test for condition; observed power $=.58$ ) and would have probably reached significance with $\sim 5$ additional participants; future researchers might take note of this. Even though the majority of analyses employed in the present study were adequately powered, researchers and practitioners should be wary of the relatively small sample size employed $(N=27)$ when generalising the findings to the wider population of white, British undergraduate students.

\section{Recommendations for practice and future research}

The present findings have implications for practitioners; particularly, as the tasks used were analogous to those employed in gymnasia and sports conditioning sessions. Firstly, men may place more importance on the metronomic regulation of their movement and their performance can therefore be rendered less efficient by the wrong type of music (i.e., oudeterous). Hence, music used for male groups should have high motivational qualities and a particularly prominent beat. Secondly, women may be more responsive to aspects of music that influence affect during anaerobic exercise; namely the melody (tune) and the combination of notes used simultaneously, which is known as harmony (Karageorghis et al., 2006; and Karageorghis et al., 1999).

In order to further explore any possible gender differences in the synchronous response to music, tasks with a greater degree of greater choreographic element might be employed. However, this line of investigation has the potential to discriminate against males who may have little movement-to-music experience and hence be uncomfortable with the protocol. For this reason, it would be necessary to control for the effects of previous exercise-to-music exposure; a personal variable that future researchers might consider screening for.

A finding that warrants further investigation is the female affective response to music during anaerobic exercise. Future research might reveal whether this interaction effect is 
replicable under different conditions and using a range of exercise modalities. It is questionable whether the affective component of music is as significant during anaerobic exercise as it is during steadily-paced, long-duration aerobic exercise when introspection and internal dialogue are more prevalent (Callen, 1983). During anaerobic exercise the role of arousal may prove more pivotal and this should be investigated in tandem with that of affect, possibly utilizing measures based on the circumplex model of affect (e.g., the Affect Grid). Also, qualitative investigation comprising of interviews and observations may help to reveal the underlying causes of differential reactivity to music by gender in the physical activity sphere. In particular, a grounded theory approach, as has been applied recently to examine regulation of emotion in tennis (Bishop, Karageorghis, \& Loizou, 2007), might shed considerable light on how the benefits of synchronous music might be maximized.

\section{Conclusions}

The present study has advanced knowledge of the effects of synchronous music in exercise through demonstrating an interaction between music condition and gender for anaerobic performance and affect. In terms of performance, this interaction effect shows that men place greater emphasis on the metronomic regulation of movement than other musical elements. Novel findings emerged highlighting a gender influence in the affective response to music; however, the possible influence of gender on the propensity to synchronize movement with music requires more detailed investigation. At present, it appears that trained women do not perform better than trained men when synchronous movements are compared. Although motivational and oudeterous music have no influence per se on anaerobic endurance or affect during exhaustive circuit-type exercises, men and women may exhibit different patterns of affective response.

\section{References}

H.M. Alessio and K.M. Hutchinson, Effects of submaximal exercise and noise exposure on hearing loss, Research Quarterly for Exercise and Sport 62 (1991), pp. 413-419.

M.H. Anshel and D.Q. Marisi, Effects of music and rhythm on physical performance, Research Quarterly 49 (1978), pp. 109-113.

G. Atkinson, D. Wilson and M. Eubank, Effects of music on work-rate distribution during a cycling time trial, International Journal of Sports Medicine 25 (2004), pp. 611-615.

D.T. Bishop, C.I. Karageorghis and G. Loizou, A grounded theory of young tennis players' use of music, Journal of Sport \& Exercise Psychology 29 (2007), pp. 584-607.

K.E. Callen, Mental and emotional aspects of long distance running, Psychosomatics 24 (1983), pp. 133-151.

M. Clynes and J. Walker, Neurobiologic functions of rhythm time and pulse in music. In: M. Clynes, Editor, Music, mind and brain: The neuropsychology of music, Plenum Press, New York, NY (1982), pp. 171-216.

R.D. Crowther and K. Durkin, Sex- and age-related differences in musical behaviour, interests and attitudes towards music of 232 secondary school students, Educational Studies 20 (1982), pp. 13-18. 
J. Edworthy and $\mathrm{H}$. Waring, The effects of music tempo and loudness level on treadmill exercise, Ergonomics 49 (2006), pp. 1597-1610.

D. Elliott, S. Carr and D. Orme, The effect of motivational music on sub-maximal exercise, European Journal of Sport Science 5 (2005), pp. 97-106.

C.J. Hardy and J. Rejeski, Not what but how one feels: the measurement of affect during exercise, Journal of Sport \& Exercise Psychology 11 (1989), pp. 304-317.

D.J. Hargreaves and A.C. North, The social and applied psychology of music, Oxford University Press, Oxford, UK (2008).

P. Harris, Designing and reporting experiments in psychology (3rd ed.), McGraw-Hill Education, New York, NY (2008).

Y. Hayakawa, H. Miki, K. Takada and K. Tanaka, Effects of music on mood during bench stepping performance, Perceptual and Motor Skills 90 (2000), pp. 307-314.

C.I. Karageorghis, The scientific application of music in sport and exercise. In: A.M. Lane, Editor, Sport and exercise psychology, Hodder Education, London, UK (2008), pp. 109-137.

C.I. Karageorghis, K.M. Drew and P.C. Terry, Effects of pretest stimulative and sedative music on grip strength, Perceptual and Motor Skills 83 (1996), pp. 1347-1352.

C.I. Karageorghis, L. Jones and D.C. Low, Relationship between exercise heart rate and music tempo preference, Research Quarterly for Exercise and Sport 77 (2006), pp. 240250.

C.I. Karageorghis, L. Jones and D.P. Stuart, Psychological effects of music tempi during exercise, International Journal of Sports Medicine 29 (2008), pp. 613-619.

C.I. Karageorghis, D.A. Mouzourides, D.L. Priest, T.A. Sasso, D.J. Morrish and C.L. Whalley, Psychophysical and ergogenic effects of synchronous music during treadmill walking, Journal of Sport \& Exercise Psychology 31 (2009), pp. 18-36.

C.I. Karageorghis, D.L. Priest, P.C. Terry, N.L.D. Chatzisarantis and A.M. Lane, Redesign and initial validation of an instrument to assess the motivational qualities of music in exercise: the Brunel Music Rating Inventory-2, Journal of Sports Sciences 24 (2006), pp. 899-909.

C.I. Karageorghis and P.C. Terry, The psychophysical effects of music in sport and exercise: a review, Journal of Sport Behavior 20 (1997), pp. 54-68.

C.I. Karageorghis and P.C. Terry, The psychological, psychophysical and ergogenic effects of music in sport: a review and synthesis. In: A.J. Bateman and J.R. Bale, Editors, Sporting sounds: Relationships between sport and music, Routledge, London, UK (2009), pp. 13-36.

C.I. Karageorghis, P.C. Terry and A.M. Lane, Development and validation of an instrument to assess the motivational qualities of music in exercise and sport: the Brunel Music Rating Inventory, Journal of Sports Sciences 17 (1999), pp. 713-724. 
G. Keppel and T.D. Wickens, Design and analysis: A researcher's handbook (4th ed.), Prentice Hall, Englewood Cliffs, NJ (2004).

E.W. Large, On synchronizing movements to music, Human Movement Science 19 (2000), pp. 527-566.

M.R. Leary and R.M. Kowalski, The self-presentation model of social phobia. In: R.G. Heimberg, M.R. Liebowitz, D.A. Hope and F.R. Schneier, Editors, Social phobia: Diagnosis, assessment, and treatment, Guilford Press, New York, NY (1995), pp. 94112.

C. Lee and P.J. Clough, The influence of rhythm and personality in the endurance response to motivational asynchronous music, Journal of Sports Sciences 24 (2006), pp. 187-195.

D.L. Priest, C.I. Karageorghis and N.C.C. Sharp, The characteristics and effects of motivational music in exercise settings: the possible influence of gender, age, frequency of attendance, and time of attendance, Journal of Sports Medicine and Physical Fitness 44 (2004), pp. 77-86.

W.J. Rejeski, Perceived exertion: an active or passive process?, Journal of Sport Psychology 75 (1985), pp. 371-378.

M. Roerdink, Anchoring: Moving from theory to therapy, IFKB, Amsterdam, Netherlands (2008).

B. Shen, A. Chen, K. Scrabis and H. Tolley, Gender and interest-based motivation in learning dance, Journal of Teaching in Physical Education 22 (2003), pp. 396-409

S. Simpson and C.I. Karageorghis, The effects of synchronous music on 400-m sprint performance, Journal of Sports Sciences 24 (2006), pp. 1095-1102.

F.L. Smoll and R.W. Schultz, Accuracy of rhythmic motor behavior in response to preferred and nonpreferred tempos, Journal of Human Movement Studies 8 (1982), pp. 123-128.

B.G. Tabachnick and L.S. Fidell, Using multivariate statistics (5th ed.), Allyn and Bacon, Needham Heights, MA (2007).

G. Tenenbaum, A social-cognitive perspective of perceived exertion and exercise tolerance. In: R.N. Singer, H.A. Hausenblas and C. Janelle, Editors, Handbook of sport psychology, Wiley, New York, NY (2001), pp. 810-822.

F.R. Wilson, Tone deaf and all thumbs?, Viking, New York, NY (1986).

R.J. Zatorre, A.R. Halpern, D.W. Perry, E. Meyer and A.C. Evans, Hearing in the mind's ear: a PET investigation of musical imagery and perception, Journal of Cognitive Neuroscience 8 (1996), pp. 29-46.

\section{Acknowledgement}

The authors would like to acknowledge the contribution of Chezarne Williams in the data collection phase of this study. 